\title{
Visualization of Facial Attractiveness Factors Using Gradient-Weighted Class Activation Mapping
}

\author{
Takanori SANO* \\ * Kozo Keikaku Engineering Corporation, 4-5-3 Chuo, Nakano-ku, Tokyo 164-0011, Japan \\ takanori-sano@kke.co.jp
}

\begin{abstract}
Research on attractiveness prediction using facial features has been actively conducted in recent years. The use of convolutional neural networks (CNNs) has been reported to achieve highly accurate predictions. In this study, we used Gradient-Weighted Class Activation Mapping (Grad-CAM) to visualize the hidden layers of the constructed CNN model, which enabled us to investigate the attractiveness factors of faces and to confirm the features that are important for prediction. The results showed that this model has important features in the area related to sexual dimorphism, which is consistent with the findings of psychological research.
\end{abstract}

Keywords: Deep Learning, Convolutional Neural Network, Gradient-Weighted Class Activation Mapping, Psychology

\section{INTRODUCTION}

Although it has long been believed that "Beauty is in the eye of the beholder," several studies have shown that facial attractiveness is highly consistent across genders and cultures [1]. It has been reported that facial averageness [2], symmetry [3], and sexual dimorphism [3] are important factors that influence the perception of facial attractiveness in humans, but the details of facial features and the psychological mechanisms that affect the perception of attractiveness are still unclear.

In the field of machine learning, considerable research on attractiveness prediction using facial features has been conducted in recent years [4,5]. Facial attractiveness prediction has been shown to be useful in applications such as make-up recommendation [6]. Research using convolutional neural networks (CNNs) has been reported to achieve a high prediction accuracy [7]. In addition, some studies have discussed the features that are important for attractiveness prediction by visualizing the hidden layers of the constructed CNN model. For example, Xu et al. (2015) [8] developed a facial attractiveness prediction model using a $\mathrm{CNN}$ and visualized the hidden layers and extracted the facial contours, eyes, and mouth as important features for predicting the facial attractiveness. This is consistent with the intuition of human visual recognition. Therefore, using a prediction model, such as a CNN, it is possible to extract universal features for facial attractiveness. However, most of the previous studies on feature extraction via hidden layer visualization are aimed at investigating methods of improving the prediction accuracy, and sufficient research has not been conducted to understand the facial features that affect the human attractiveness perception or the attractiveness perception mechanism.

\section{PURPOSE}

In this study, we used a CNN to develop an attractiveness prediction model and visualize the hidden layers. This research was aimed at extending the understanding of facial features that affect the human attractiveness perception and attractiveness perception mechanisms by investigating the similarities and differences between the features that the model determines as important and the findings of psychological research.

\section{PROCEDURE}

The validation was performed using a benchmark database, SCUT-FBP5500 [9], which contains 2750 male and 2750 female face images with attractiveness scores labeled by 60 raters.

\subsection{Model prediction experiment}




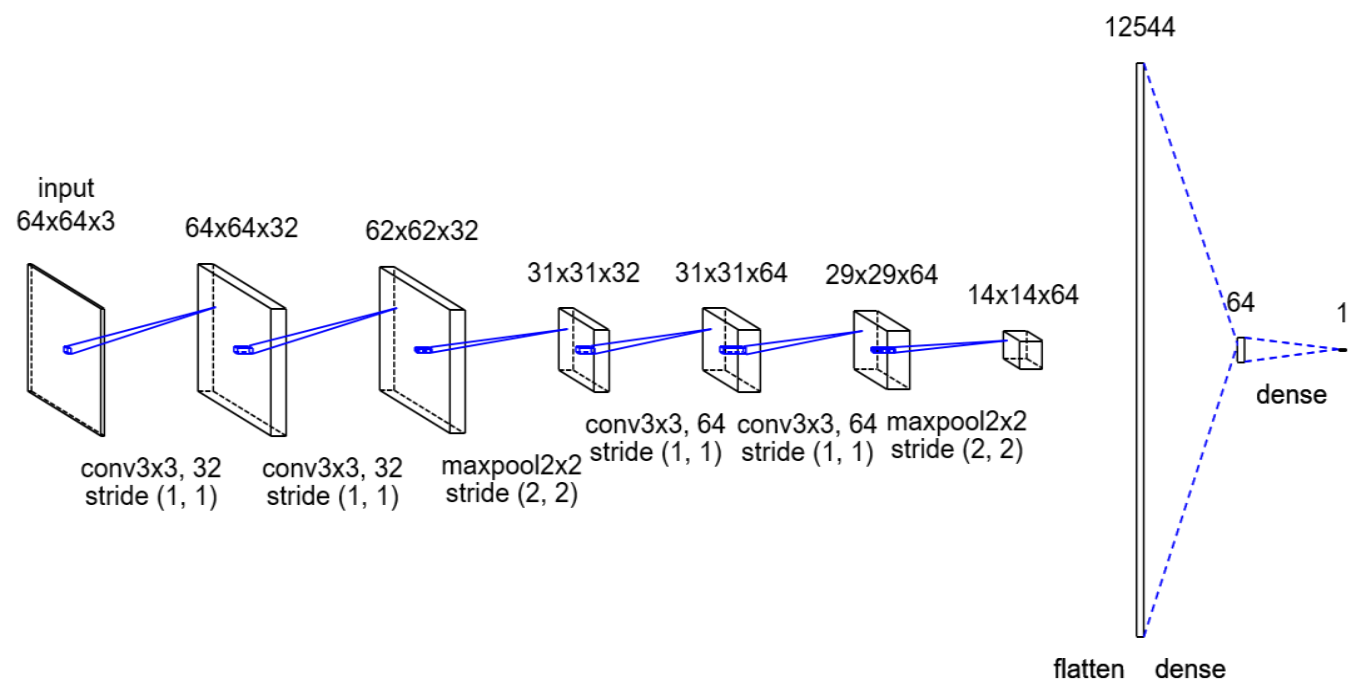

Figure 1: Model networks

Table 1: Correlation coefficient results

\begin{tabular}{|c|c|c|c|c|c|c|c|}
\hline model & sex & 1 & 2 & 3 & 4 & 5 & average \\
\hline \multirow{2}{*}{ CNN } & male & 0.753 & 0.798 & 0.750 & 0.750 & 0.806 & 0.771 \\
\cline { 2 - 8 } & female & 0.792 & 0.787 & 0.794 & 0.807 & 0.790 & 0.794 \\
\hline \multirow{2}{*}{ SVR } & male & 0.743 & 0.730 & 0.696 & 0.676 & 0.672 & 0.704 \\
\cline { 2 - 8 } & female & 0.702 & 0.715 & 0.678 & 0.713 & 0.707 & 0.703 \\
\hline \multirow{2}{*}{ GPR } & male & 0.386 & 0.340 & 0.388 & 0.361 & 0.304 & 0.356 \\
\cline { 2 - 8 } & female & 0.342 & 0.314 & 0.361 & 0.369 & 0.386 & 0.354 \\
\hline \multirow{2}{*}{ LR } & male & 0.234 & 0.167 & 0.279 & 0.286 & 0.160 & 0.225 \\
\cline { 2 - 8 } & female & 0.206 & 0.155 & 0.249 & 0.185 & 0.197 & 0.199 \\
\hline
\end{tabular}

In the first experiment, we tested the accuracy of the attractiveness prediction model. Although the study conducted by Xu et al. (2015) [8] only used the female face dataset, we validated the results on each of the male and female face datasets in this study to compare the differences between the genders; we split the male and female images into separate datasets for validation. In the validation process, 2200 images were randomly selected from the 2750 images to generate the training dataset, and the rest were used as the test dataset. To reduce the bias because of data partitioning, all the verifications were performed with a five-part cross-validation. Following previous studies [10,11], the Pearson correlation between the image label values and the prediction results was used as the metric to evaluate the prediction performance. In addition, to compare our CNN model with existing methods in terms of the prediction accuracy, we used linear regression (LR), Gaussian process regression (GPR), and support vector regression (SVR) as benchmark models for machine learning. The prediction accuracy of each method was calculated under the same conditions.

\subsection{Visualization experiment}

In the next experiment, we visualized the hidden layer using the developed model. We explored the similarities and differences between the visualization results and the findings of psychological research and discussed the facial features for attractiveness perception.

\section{MODEL}

The network diagram of the model used in this study is shown in Figure 1.

The number above each layer in the figure indicates the size of the layer, and the description below each layer indicates the processing in that layer. The Dropout process is performed immediately after each pooling and dense layer. ReLU was used as the activation function, the mini-batch size was fixed at 64, and training was performed with 1000 epochs. 


\section{MODEL PREDICTION RESULT}

The CNN model was constructed, and the accuracy was verified. We achieved average correlations of 0.771 and 0.789 with respect to the test data on the male and female face datasets, respectively. The prediction accuracies of LR, GPR, and SVR, which are benchmark models for machine learning, were $0.225,0.356$, and 0.704 for the male face dataset and 0.199 , 0.354 , and 0.703 for the female face dataset, respectively. Our model outperformed previous machine learning methods in terms of accuracy. The results are presented in Table 1.

\section{VISUALIZATION RESULT}

Using the model trained on all the data, we visualized the hidden layer closest to the output layer using gradient-weighted class activation mapping (Grad-CAM) [12], which is excellent for visualizing the basis of prediction decisions in a CNN, to observe the features based on which the model was predicting attractiveness values. In this study, to understand the major tendency of visualization, we excluded the images calculated by Grad-CAM for each male and female images, where the mean value of the image heat map was less than $10 \%$ of the distribution of all images. Next, the top 50 images with the highest attractiveness score label value for each male and female image were extracted and averaged by overlaying the face images (Fig. 2, 3). The results indicate that the male images tended to show activation around the eyebrows, whereas the female images tended to show activation under and around the eyes.

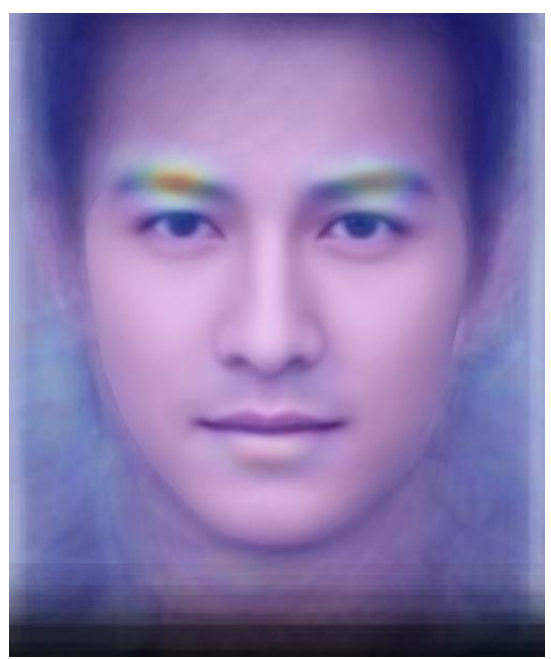

Figure 2: Result of visualization of averaged male images using Grad-CAM.

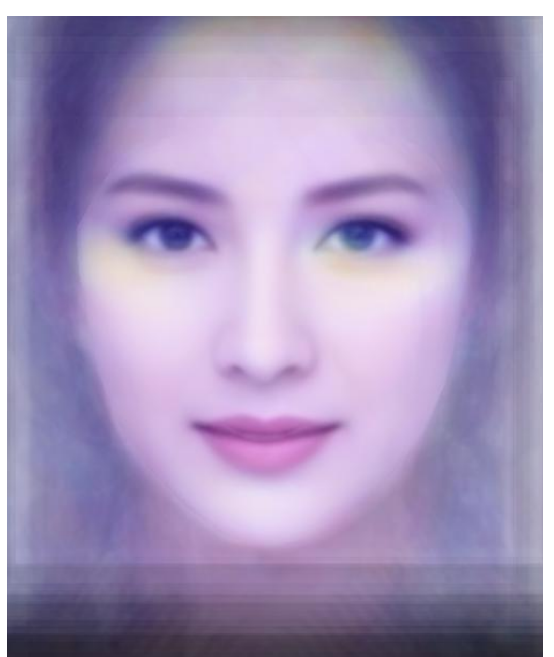

Figure 3: Result of visualization of averaged female images using Grad-CAM.

\section{DISCUSSION}

Based on previous studies, it is generally known that sexual dimorphism and facial attractiveness are correlated [3]. Russel (2009) [13] showed that luminance contrast between facial features and the skin is significantly correlated with sexual dimorphism. Jones et al. (2015) [14] proposed that men have a higher luminance contrast than women do in the eyebrow region, and women have a higher luminance contrast than men do in the eye region. The contrast was proposed to be higher in the eyebrow region than in the eye region. In addition, they reported that women use make-up to accentuate the features of the eye region. It is possible that the model constructed in this study also focuses on features that emphasize sexual dimorphism around the eyebrows and eyes. This result supports the view proposed by Russel (2009) [13] and Jones et al. (2015) [14] with regard to machine learning.

As shown earlier, the features extracted from the model by visualizing the hidden layer have some similarities with the findings of psychological research. In this study, we focused on the images with high attractiveness scores. In the future, 
more detailed analysis will be possible by considering the differences in the intensity of attractiveness and the prediction accuracy of the model for each image.

\section{CONCLUSION}

Using the CNN model, we achieved accuracies of 0.771 and 0.794 (Pearson correlation) on the male and female face datasets, respectively, for facial attractiveness prediction. These accuracies are higher than those of benchmark machine learning models. In addition, the features extracted from the model by visualizing the hidden layers showed some similarities with the findings of psychological research. The male images tended to show activation around the eyebrows, whereas the female images tended to show activation under and around the eyes. The approach discussed herein can help us in understanding the facial features that affect the perception of human attractiveness. In the future, the consideration of the model processing will help in elucidating the psychological mechanism of attractiveness perception.

\section{REFERENCES}

[1] J. Langlois, L. Kalakanis, A. Rubenstein, A. Larson, M. Hallam and M. Smoot; Maxims or myths of beauty?: A meta-analytic and theoretical review, Psychological Bulletin; 126, pp.390-423, 2000.

[2] D.I. Perrett, K.A. May and S. Yoshikawa; Facial shape and judgments of female attractiveness: Preferences for non-average characteristics, Nature; 386, pp.239-242, 1994.

[3] S.W. Gangestad, R. Thornhill and C.E. Garver-Apgar; Men's facial masculinity predicts changes in their female partners' sexual interests across the ovulatory cycle, whereas men's intelligence does not, Evolution and Human Behavior; 31, pp.412-424, 2010.

[4] H. Mao, L. Jin and M. Du; Automatic classification of Chinese female facial beauty using Support Vector Machine, In Proceedings of IEEE International Conference on Systems, Man and Cybernetics; pp.4842-4846, 2009.

[5] D. Zahang, Q. Zhao and F. Chen; Quantitative analysis of human facial beauty using geometric features, Pattern Recognition; 44(4), pp.940-950, 2011.

[6] L. Liang, L. Jin and X. Li; Facial skin beautification using adaptive region-aware mask, IEEE Transactions on Cybernetics; 44(12), pp.2600-2612, 2014.

[7] J. Xu, L. Jin, L. Liang, Z. Feng, D. Xie and H. Mao; Facial attractiveness prediction using psychologically inspired convolutional neural network (pi-cnn), In IEEE International Conference on Acoustics, Speech and Signal Processing; pp.1657-1661, 2017.

[8] J. Xu, L. Jin, L. Liang, Z. Feng and D. Xie; A new humanlike facial attractiveness predictor with cascaded fine-tuning deep learning model; arXiv:1511.02465; 2015.

[9] L. Liang, L. Lin, L. Jin, D. Xie and M. Li; SCUT-FBP5500: A diverse benchmark dataset for multi-paradigm facial beauty prediction, In ICPR; pp.1598-1603, 2018.

[10] W. Chiang, H. Lin, C. Huang, L. Lo and S. Wan; The cluster assessment of facial attractiveness using fuzzy neural network classifier based on 3D Moir features, Pattern Recognition; 47(3), pp.1249-1260, 2014.

[11] H. Yan; Cost-sensitive ordinal regression for fully automatic facial beauty assessment, Neurocomputing; 129 , pp.334-342, 2014.

[12] R. Selvaraju, M. Cogswell, A. Das, R. Vedantam, D. Parikh and D. Batra; Grad-CAM: Visual explanations from deep networks via gradient-based localization. In arXiv preprint arXiv:1610.02391; pp.1-24, 2016.

[13] R. Russell; A sex difference in facial contrast and its exaggeration by cosmetics, Perception; 38, pp.1211-1219, 2009.

[14] A.L. Jones, R. Russell and R. Ward; Cosmetics alter biologically-based factors of beauty: Evidence from facial contrast, Evolutionary Psychology; 13, pp.210-229, 2015. 